\title{
Yellow nail syndrome and xanthogranulomatous pyelonephritis
}

\author{
HD Danenberg, R Eliashar, G Flusser, E Rosenmann, T Chajek-Shaul
}

\begin{abstract}
Summary
We report a case of a 74-year-old woman exhibiting the yellow nail syndrome in association with ipsilateral xanthogranulomatous pyelonephritis. Nephrectomy resulted in complete resolution of the pleural effusion and slow improvement of the yellow nails. This report calls attention to the renal-related pleural effusion, and to the thorough investigation needed for the yellow nail syndrome.
\end{abstract}

Keywords: yellow nail syndrome, pleural effusion, xanthogranulomatous pyelonephritis

\section{Introduction}

The yellow nail syndrome is a combination of yellow discoloured nails, lymphoedema and a pleuro-pulmonary disorder, mainly pleural effusion. Most cases reported are idiopathic and the prognosis is benign. However, the syndrome has been described in association with malignancy ${ }^{1}$ and various other disorders, necessitating a thorough evaluation. Whenever an obvious cause for pleural effusion is not discernible after initial evaluation, a renal aetiology should also be considered. ${ }^{2}$ We report, for the first time to the best of our knowledge, a case of yellow nail syndrome associated with ipsilateral xanthogranulomatous pyelonephritis.

\section{Case report}

A 74-year old woman was admitted for evaluation of unremitting left-sided exudative pleural and pericardial effusion. A definite aetiology had not been found during extensive evaluations over 18 months, including pleural and pericardial biopsies and repeated cultures for tuberculosis. The effusion did not resolve despite recurrent pleurocenteses. Non-insulin dependent diabetes and hypertension had been present for years and she was treated with metformin, clonidine and nifedipine, respectively. On examination vital signs were normal. Examination of the chest revealed dullness on percussion, with decreased fremitus and breathing sounds in the lower half of the left lung. Heart sounds were diminished. Yellow nails, and $4 \mathrm{~mm}$ pitting oedema over the lower limbs were present. No other physical findings were found. On urinalysis multiple leukocytes and Gram-negative rods were seen. Urine culture grew Proteus mirabilis (20 000 colonies/ $\mathrm{ml})$. Erythrocyte sedimentation rate was $120 \mathrm{~mm}$ in the first hour. Haemoglobin, $11.2 \mathrm{~g} /$ dl with mean corpuscular volume $77 \mathrm{fl}$, white blood cell count $15.5 \times 10^{9} / 1$ and $511 \times 10^{9} / 1$ platelets. Biochemistry was normal. Chest Xray showed a large left-sided pleural effusion and mild enlargement of the cardiac silhouette. Protein content of the pleural fluid was $49 \mathrm{~g} / \mathrm{l}$, with a high lactate dehydrogenase level of 2609 U (normal 300-620 U). Large numbers of polymorphonucleocytes were present, but no bacteria or malignant cells were seen. Repeated bacterial cultures were negative. Computed tomographic (CT) scan of the chest demonstrated a left-sided pleural effusion, with no pulmonary pathology. An echocardiogram showed a small amount of pericardial effusion with no evidence of tamponade. On CT scan of the abdomen (figure) a large hydronephric left kidney with staghorn calculus, and paranephric abscesses invading peri-renal structures were found. An intravenous pyelogram showed no secretion from the left kidney. The patient was referred for nephrectomy. Microscopical examination of the kidney revealed chronic pyelonephritis with abscess formation and palisading granuloma with numerous foam cells, features of xanthogranulomatous pyelonephritis. Following surgery prompt resolution of the pleural effusion was seen. Five months after the operation an obvious decrease in yellow colour of the nails was also observed.

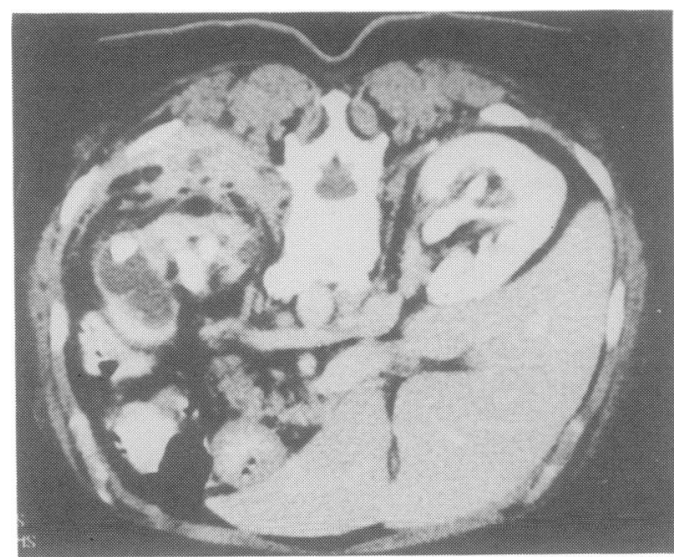

Figure Axial computed tomography of the abdomen demonstrating a large hydronephric left kidney with stones and abscesses invading extra-renal structures 


\section{Discussion}

Yellow nail syndrome, was first described in 1964 by Samman and White, ${ }^{3}$ consists of the triad yellow discoloured nails, lymphoedema, and pleural effusion. The pleural effusion is usually exudative, either idiopathic or secondary to infection, or chylothorax. Other kinds of respiratory involvement have been reported in conjunction with yellow nails. ${ }^{4}$ Disappearance of the yellow discolouration has been noted either spontaneously or following resolution of the pulmonary disease. ${ }^{5,6}$ In most cases of yellow nail syndrome reported up to now no extramediastinal aetiology has been described.

The pathogenic mechanism underlying the yellow nail syndrome has not been defined. Abnormality of lymphatic vessels was suggested as the cause of the syndrome by Samman and White in their original description of the syndrome. ${ }^{3}$ The theory is supported by lymphangiographic findings which in most patients showed few, hypoplastic or dilated, deficient lymphatics. ${ }^{1}$ Electron microscopy in two cases ${ }^{1,7}$ noted dilated but otherwise normal lymphatics.

The association of renal infection with ipsilateral pleural effusion was previously described. ${ }^{8,9}$ Of interest is a special variant of chronic urinary infection, xanthogranulomatous pyelonephritis, which is a destructive inflammatory process accompanied by pyelonephritis that may be complicated by fistula formation connecting the adjacent destroyed kidney and the pleural space. ${ }^{10,11}$ The diagnosis of xanthogranulomatous pyelonephritis is mainly histologic, and was supported by radiological findings in the above case.

1 Nordkild P, Kromann-Andersen H, Struve-Christensen E. Yellow nail syndrome - the triad of yellow nails, lym phedema and pleural effusions. Acta Med Scand 1986; 219 $221-7$

2 Sahn SA, Miller KS. Obscure pleural effusion. Look to the kidney. Chest 1986; 90: 631 .

3 Samman PD, White WF. The 'yellow nail' syndrome. $\mathrm{Br} f$ Dermatol 1964; 76: 153-7.

4 Venecie PY, Dicken CH. Yellow nails syndrome: report of five cases. $\mathcal{F}$ Am Acad Dermatol 1984; 10: 187-92.

5 Pavlidakev GP, Hashimoto K, Blum D. Yellow nails syndrome. F Am Acad Dermatol 1984; 11: 509-12.

6 Ben-Yehuda A, Ben-Chetrit E, Eliakim M. Yellow nail syndrome: case report and review of the literature. Isr $\mathfrak{F} \mathrm{Med}$ $S c i$ 1986; 22: report

7 Solal-Celigny P, Cormier Y, Purnier M. The yellow nail syndrome. Light and electron microscopy aspects of the pleura. Arch Pathol Lab Med 1983; 107: 183-5.
Yellow nail syndrome: features

- yellow nails

- pleuropulmonary symptoms (pleural effusion, chronic bronchitis, bronchiectasis,

pneumonia, tuberculosis, sinusitis)

- lymphoedema

\section{Yellow nail syndrome: associations}

- malignant disease (malignant melanoma, carcinoma of the larynx, lung and breast, Waldenstrom's macroglobulinaemia, Hodgkin's lymphoma)

- thyroid disease (Hashimoto's thyroiditis, hypothyroidism, thyrotoxicosis)

- hypogammaglobulinaemia

- rheumatoid arthritis

- recurrent erysipelas

In our patient reported above the correct diagnosis was not easily achieved. However, when chronic pyelonephritis and perinephric abscesses were discovered, surgery resulted in prompt resolution of the pleural effusion followed by slow resolution of the yellow discolouration of the nails.

In summary, we report a patient with yellow nail syndrome associated with homolateral xanthogranulomatous pyelonephritis. Nephrectomy resulted in resolution of the clinical picture. We conclude that an extramediastinal aetiology should at times be considered both for the yellow nail syndrome and exudative pleural effusion of unknown aetiology.

8 Buisseret PD. Pleural effusion secondary to perinephric abscess. Lancet 1982; 2: 100 .

9 Patel NP, Pitts WR, Ward JN. Solitary infected renal cyst: report of 2 cases and review of the literature. Urology 1978; 11: $164-7$.

10 Parsons MA, Harris SC, Grainger RG, Ross B, Smith JA, Williams JL. Fistula and sinus formation in xanthogranulomatous pyelonephritis. A case report and review of four cases. Br $\mathcal{F}$ Urol 1986; 58: 488-93.

11 Fernandez-Garcia JS, Garcia-Aguayo FJ, Llopis-Cartagena M, Carratala-Torregrosa JA, Botella-Almodovar $\mathbf{R}$ Renopleural fistula secondary to xanthogranulomatous pyelonephritis. Acta Urol Esp 1989; 13: 45-8. 